\title{
Cólera en Bolívar de 1991 a 1997
}

\author{
Delfina Urbina ${ }^{1}$, Ketty Mendoza ${ }^{1}$, Marta. Puello ${ }^{1}$, Norma Baena ${ }^{2}$, Gregorio Young ${ }^{1}$, \\ Luis A. Flechas ${ }^{1}$, William Pomares ${ }^{2}$
}

\begin{abstract}
Resumen
El cólera, que se ha presentado a través de los siglos en forma endémica y epidémica, continúa siendo un problema de salud pública. Desde 1991, cuando el cólera apareció por primera vez en nuestro país en este siglo, el departamento de Bolívar ha sido uno de los más afectados en la costa atlántica. A partir de entonces, las autoridades de salud del departamento (Dasalud) solicitaron apoyo tecnológico para el diagnóstico microbiológico y capacitación del recurso humano al Laboratorio del Posgrado de Microbiología de la Universidad de Cartagena. Se adelantó este trabajo descriptivo de cooperación interinstitucional con el propósito de analizar el comportamiento del cólera en los municipios de Bolívar. A partir del caso índice, se inició la recolección de muestras en pacientes sospechosos provenientes de las cinco regionales de Dasalud. El diagnóstico de la enfermedad se hizo con base en los criterios clínicos, microbiológicos y epidemiológicos establecidos. Las heces recolectadas en el medio de transporte fueron procesadas para su aislamiento e identificación en medios de cultivos bacteriológicos, bioquímicos y serotipificación con antisueros específicos.
\end{abstract}

De septiembre de 1991 al octavo período de 1997, se informaron 3.470 casos en 38 de 42 municipios y $94,6 \%$ ocurrió en las regionales 1 y $3 ; 60,2 \%$ correspondió a varones, $39,8 \%$ a mujeres y $79,2 \%$ fueron mayores de 14 años; hubo 33 defunciones; $39,8 \%$ de los casos fue diagnosticado microbiológicamente, con el hallazgo de Vibrio choleraeO1, biotipo EI Tor, serotipos Inaba y Ogawa. V. cholerae O1, serotipo Ogawa, presente en el brote de 1995 desplazó al serotipo Inaba predominante en 1991.

En 1991, el número de casos fue nueve veces mayor que en el brote de 1995 y, en 1997, aumentó $45,2 \%$ con relación a los dos últimos años; la letalidad, a su vez, mostró índices preocupantes a partir de 1992.

Los municipios más afectados fueron: Cartagena, Mahates, Montecristo, María la Baja, Pinillos, Achí, Magangué, Arjona, Turbana, Calamar, Mompox, Santa Catalina, Hatillo de Loba, Turbaco, San Martín de Loba, Carmen de Bolívar, Villanueva, Zambrano, Morales, Talaigua Nuevo y Río Viejo, entre otros.

\section{Summary}

Cholera, which has been present throughout the centuries in an endemic and epidemic form, continues to be a public health problem. Since 1991, when cholera appeared in our country for the first time in this century, the Bolivar department has been one of the most affected on Colombia's Atlantic coast.

Laboratorio del Posgrado de Microbiología, Universidad de Cartagena, Cartagena, Bolívar.

2 Departamento Administrativo de Salud de Bolivar (Dasalud), Cartagena, Bolívar. 
At that time the department's health authority (Dasalud) asked the University of Cartagena's Postgraduate Microbiology Laboratory for technological help in microbiological diagnosis and human resource training.

This work, describing inter-institutional cooperation was thus brought into effect with the intention of analysing cholera behaviour in Bolívar's municipalities.

From the index case, suspected patient sample collection was begun in Dasalud's five regions. Diagnosis of the disease was based on established clinical, microbiological and epidemiological criteria. Faecal samples, being the means of disease transmission, were processed for isolation and identification in bacteriological and biochemical culture media and for serotyping with specific antisera.

From September 1991 to the eighth epidemiological period of 1997, 38 of the 42 municipalities reported 3,470 cases. $94.6 \%$ occurred in region 1 and $3 ; 60.2 \%$ occurred in men and $39.8 \%$ in women; $79.2 \%$ were older than 14 years of age; there were 33 deaths. Vibrio cholerae O1 biotype EI Tor serotypes Inaba and Ogawa was the microbiological diagnosis in $39.8 \%$ of the cases. Vibrio cholerae O1 serotype Ogawa, present in the 1995 outbreak, displaced the Inaba serotype which had prevailed in 1991.

In 1991 the number of cases was nine times greater than in the 1995 outbreak. In 1997 there was a $45.2 \%$ increase in relation to the last two years. Mortality, in its turn, has shown worrying indices from 1992 onwards.

The most affected municipalities were: Cartagena, Mahates, Montecristo, María la Baja, Pinillos, Achí, Magangué, Arjona, Turbana, Calamar, Mompox, Santa Catalina, Hatillo de Loba, Carmen de Bolívar, Villanueva, Zambrano, Morales, Talaigua Nuevo and Río Viejo, amongst others.

El cólera, enfermedad reemergente, se ha mantenido endémico y latente en India por varios siglos desde 1817. En 1828, se extendió por primera vez más allá de las fronteras de ese país, invadiendo a Europa $(1,2)$.

En 1832, llegó a las costas americanas procedente del Viejo Mundo en barcos que llegaban a Canadá y penetró así al interior del país; simultáneamente apareció en Estados Unidos (2).

Durante la segunda pandemia, ocurrida entre 1833 y 1850 , el cólera procedente de Panamá afectóa Suramérica, el Caribe, Centroamérica y, nuevamente, a Nueva York y California; de allí se expandió a otras ciudades costeras y al sur de este país. En Suramérica, penetró a Colombia y Ecuador alcanzando a Bogotá y Quito $(3,4)$.

Durante la tercera, cuarta y quinta pandemia, ocurridas de 1852 a 1886, se informaron casos en distintas ciudades de Norteamérica, Centroamérica, Suramérica e islas del Caribe, con la aparición de algunos brotes simultáneos (3-5).
En la sexta pandemia, ocurrida entre 1889 y 1923 , el cólera no alcanzó las Américas $(3,4)$; sin embargo, tanto en la actual pandemia como en las anteriores, hubo brotes de la enfermedad en países africanos, asiáticos y del oriente medio $(1,5)$.

La séptima y actual pandemia se inició en Sulawesi, Indonesia, en 1961. En los años 70, se presentaron casos en Pakistán e India por los movimientos masivos durante la guerra; se señala, además, que hubo brotes en Italia y Portugal. Para esa misma época, apareció el cólera nuevamente en Estados Unidos (1, 5).

Desde 1883 hasta 1937, todas las epidemias conocidas de cólera se debieron a $V$. cholerae 01 biotipo clásico. El biotipo El Tor apareció en forma virulenta en Indonesia (1). Ambos biotipos, el clásico y El Tor, han coexistido siempre en Bangladesh y durante los brotes informados a principio de los años noventa en áreas del centro y norte de esa zona, las cepas emergentes de $V$. cholerae O1 biotipo El Tor han desplazando a $V$. cholerae O139, el cual fue aislado y caracterizado en 1992 
$\mathrm{y}$, actualmente, se considera, con $\mathrm{V}$. cholerae $\mathrm{O} 1$, agente etiológico del cólera epidémico $(6,7)$.

Durante la actual pandemia, en 1991, surgió una epidemia en Perú confirmándose que los casos fueron causados por $V$. cholerae O1, biotipo El Tor, serotipo Inaba; a partir de entonces, surgieron otros casos en Suramérica (2-4).

En Colombia, el primer caso de cólera de esta séptima pandemia ocurrió el 8 de marzo de 1991 en un hombre adulto procedente de Tumaco cerca a la frontera colombo-ecuatoriana (8) y fue causado por $V$. cholerae O1, biotipo EI Tor, serotipo Inaba, y confirmado dos días después en el Instituto Nacional de Salud $(9,10)$; se informa, además, que antes del caso citado no se había diagnosticado esta enfermedad en nuestro país en el presente siglo $(11,12)$.

En los meses siguientes, se presentaron nuevos focos de la enfermedad en la costa pacífica, en Leticia, ciudad fronteriza con el Perú, extendiéndose a Tolima, el Urabá antioqueño y la costa atlántica $(2,8)$.

En esta región norte del país, el primer caso de cólera se informó en el departamento de Bolívar el 9 de septiembre de 1991. Ocurrió en un niño de 11 años procedente de Bocacerrada, Sucre, quien fue remitido al siguiente día por el médico rural del Centro de Salud de Pasacaballos al Hospital Universitario de Cartagena, donde falleció un día después. El diagnóstico de Vibrio chloerae O1, biotipo El Tor, serotipo Inaba, de este paciente se hizo en el Laboratorio del Posgrado de Microbiología de la Universidad de Cartagena y fue confirmado posteriormente por el Instituto Nacional de Salud $(9,13)$.

Teniendo en cuenta que Bolivar ha sido uno de los departamentos de la costa atlántica más afectados por el cólera, se adelantó el presente estudio con el propósito de analizar el comportamiento de la enfermedad en los 42 municipios de las cinco regionales de salud del departamento.

\section{Materiales y métodos}

A partir del caso índice, en septiembre de 1991, las autoridades del Departamento Administrativo de Salud de Bolívar (Dasalud) solicitaron apoyo en el diagnóstico microbiológico del cólera y capacitación del recurso humano en el Laboratorio del
Posgrado de Microbiología de la Universidad de Cartagena.

La búsqueda de casos y la recolección de muestras en la población de estudio fue hecha por el personal de Dasalud en el área de influencia de 42 municipios que conforman las cinco regionales del departamento, cuya población informada es de 1'843.630 habitantes (14). Las regionales de saiud, denominadas $1,2,3,4$ y 5 , están constituidas por 14, 6, 8, 6 y 8 municipios, respectivamente; en 38 de éstos, se informó la enfermedad.

El diagnóstico de la enfermedad se hizo con base en criterios clínicos, microbiológicos y de asociación epidemiológica. El total de pacientes incluyó: 1) los casos confirmados por coprocultivo que mostraron las características morfotípicas, bioquímicas y serotípicas de V. cholerae O1 (15); 2) los casos sospechosos de pacientes con la sintomatología descrita en la literatura $(11,16$, 17), y 3) los casos por asociación epidemiológica para lo cual se tuvieron en cuenta diez contactos con la misma sintomatología por cada paciente positivo microbiológicamente.

Una vez recolectadas las muestras de heces, 813 fueron remitidas en el medio de transporte CarryBlair al Laboratorio de Referencia Nacional del Instituto Nacional de Salud; 480 al laboratorio de referencia local del Posgrado de Microbiología de la Universidad de Cartagena y, durante el último período del estudio, 96 muestras al Laboratorio de Dasalud; todas las cepas fueron confirmadas en el INS.

En el aislamiento e identificación de $V$. cholerae O1, se utilizó la metodología descrita en el Manual para el diagnóstico de V. cholerae O1 (15) y otros procedimientos de rutina que se utilizan en nuestro laboratorio, los cuales se señalan a continuación.

Del medio de transporte Carry-Blair (Oxoid), las muestras se sembraron en agua peptonada al $1 \%$ por 6 horas, a $37^{\circ} \mathrm{C}$; seguidamente, se hizo nueva siembra en superficie de agar TCBS (Ref. CM 333 , Oxoid) y se incubaron a $37^{\circ} \mathrm{C}$ durante 18 a 24 horas.

A las colonias sospechosas de color amarillo brillante, bordes lisos, aspecto mucoide, planas, di- 
fíciles de coger con el asa y fermentadoras de la sacarosa, se les hizo la prueba de la cuerda.

Esta prueba consistió en suspender una colonia bacteriana con una gota de desoxicolato de sodio al $0,5 \%$ en un portaobjeto, mezclándola homogéneamente hasta obtener una suspensión viscosa que se hacía visible al levantar el asa a manera de un hilo que se engrosaba segundos después, lo que indicaba ruptura del ADN bacteriano (15).

Para la identificación bioquímica, las colonias puras se sembraron en agar lisina hierro (LIA) (Merck, Ref. 11640), agar triple azúcar hierro (TSI) (Merck, Ref. 3915), medio de SIM (Merck, Ref. 5470), caldo MRVP (Merck, Ref. 5712) y agar citrato de Simons (Merck, Ref. 2501). La prueba de oxidasa se hizo con Bactident oxidase (Merck, Ref. 13300) a partir del cultivo de agar sangre.

La serotipificación se hizo con antisueros polivalentes y monovalentes para identificación de V. cholerae, serotipo Inaba (Difco, Ref. 2430-47) y serotipo Ogawa (Difco, Ref. 2431-47).

Para el análisis de la letalidad en los diferentes años y en las regionales, se utilizó la prueba del chi cuadrado e indicadores de riesgo (riesgo relativo y riesgo atribuible), utilizando el paquete estadístico computarizado Epi-Info, versión 5.

\section{Resultados}

De septiembre de 1991 a julio de 1997 (semana epidemiológica $29,8^{\circ}$ período), la Sección de Epidemiología de Dasalud informó 3.470 casos de cólera ocurridos en 38 de 42 municipios del departamento de Bolívar.

En relación con el sexo y los grupos de edad, la enfermedad se presentó de la siguiente manera: $2.089(60,2 \%)$ fueron del sexo masculino y 1.381 $(39,8 \%)$ del sexo femenino; $2.748(79,2 \%)$ correspondieron a mayores de 14 años, $418(12 \%)$ entre 6 y 14 años, $213(6,1 \%)$ con edades entre 2 y 5 años y 91 (2,6\%) en menores de un año.

La situación del cólera en las cinco regionales, en los diferentes años, se presentó de la siguente manera: en la regional 1, hubo 2.891 casos en 13 de 14 municipios incluyendo el Distrito de Cartagena y $15(0,51 \%)$ defunciones; 2.535 de los casos ocurridos se presentaron durante 1991 y
1992; 72 casos los dos años siguientes y 284 en los últimos tres años (figura 1).

En todos los municipios de la regional 2, hubo 70 casos; 62 de éstos, entre 1991 y 1993, los 8 restantes ocurrieron en 1996 y 1997 (figura 1); en esta regional no hubo defunciones.

En la regional tres, todos los ocho municipios informaron casos de cólera, para un total de 395 casos; 47 ocurrieron de 1991 a 1993 y 348 entre 1996 y 1997 (figura 1); hubo, además, 14 (3,54\%) defunciones.

En los seis municipios de la regional cuatro, se presentaron 75 casos; 49 ocurrieron entre 1991 y 1993, los 26 restantes durante 1996 y 1997 (figura 1); se informaron, además, 4 defunciones.

De 39 casos informados en cinco de ocho municipios de la regional cinco, 36 se presentaron durante 1991 y 1992 y otros 3 casos entre 1996 y 1997 (figura 1); no hubo defunciones.

Del total de casos informados, $2.081(60 \%)$ fueron diagnosticados por asociación epidemiológica y $1.389(39,8 \%)$ microbiológicamente con los siguientes hallazgos: V.cholerae O1, biotipo EI Tor, serotipo Inaba, durante la epidemia de 1991 y Vibrio cholerae O1, biotipo El Tor, serotipo Ogawa, en un segundo brote que comenzó en septiembre de 1995. Otras bacterias causantes de diarrea tales como Salmonella typhi y Shigella boydifueron aisladas en algunos pacientes.

De 3.470 casos que hubo en total, ocurrieron 33 $(0,95 \%)$ muertes; 8 en 1991 , todas en la regional uno; en 1992, hubo 4, 3 en la regional uno y otra en la regional tres; no hubo defunciones en los dos años siguientes (figura 2).

Sin embargo, en 1995 ocurrieron 4 muertes todas en la regional uno; en 1996, hubo 3 fallecimientos, 2 en la regional tres y 1 en la regional cuatro $y$, en 1997, ocurrieron 14 defunciones, 11 en la regional tres y 3 en la regional cuatro (figura 2).

Hubo diferencia estadísticamente significativa entre el índice de muertes ocurridas entre la regional uno y las regionales tres y cuatro $(p<0,005 ; R r=$ $6,83)(p<0,005 ; R r=10,3)$, mas no para las regionales tres y cuatro, entre sí $(p>0,1)$.

Se encontró, además, que hubo diferencia estadísticamente significativa en el número de defuncio- 
Figura 1. Casos de cólera en regionales de Bolívar.

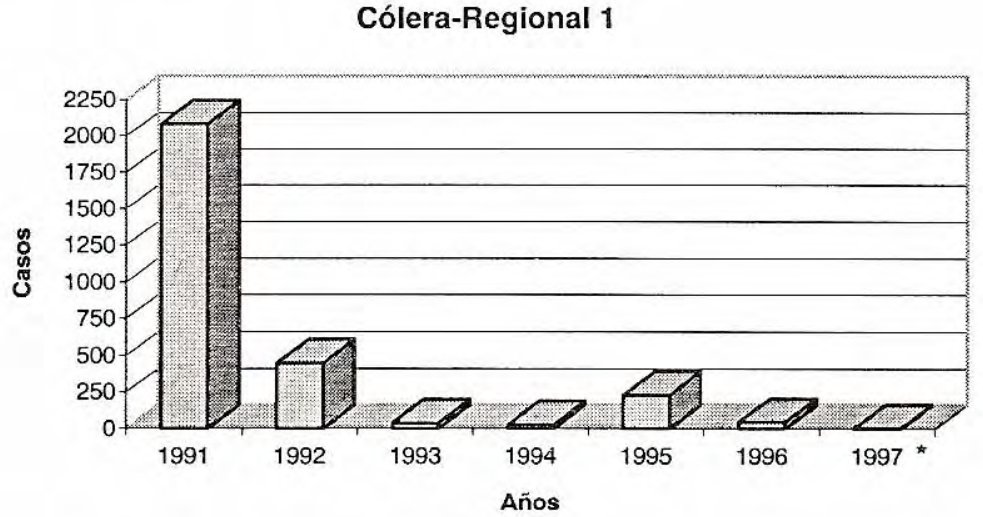

Cólera-Regional 2

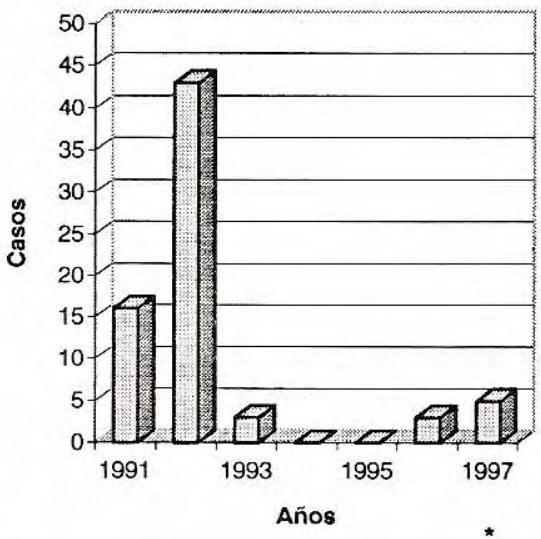

Cólera-Regional 4

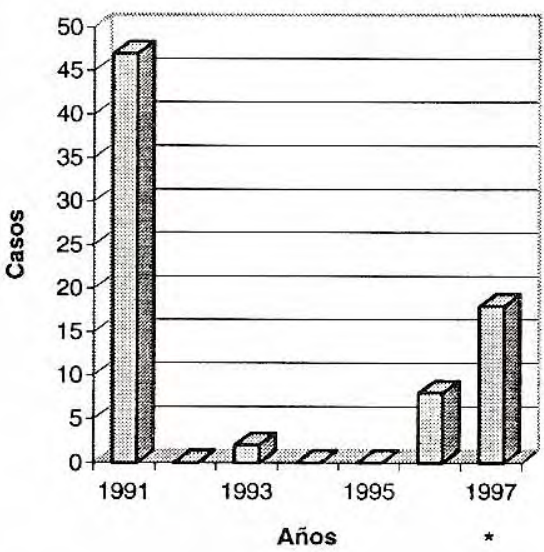

*1997:sem. epidemol 29, perìodo 80.
Cólera-Regional 3

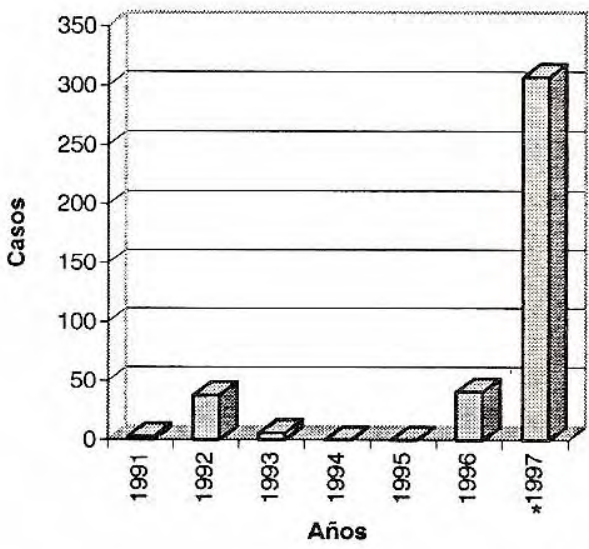

Cólera-Regional 5

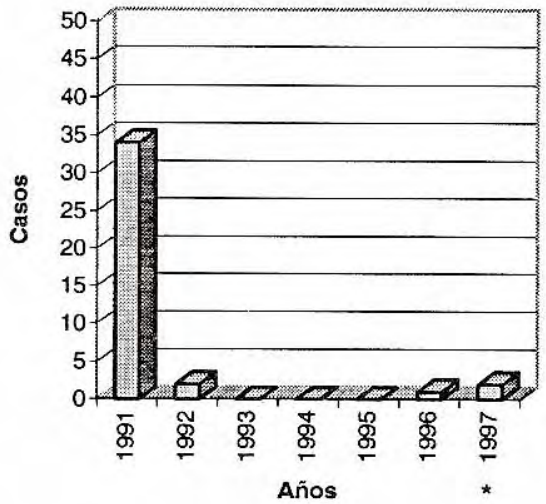




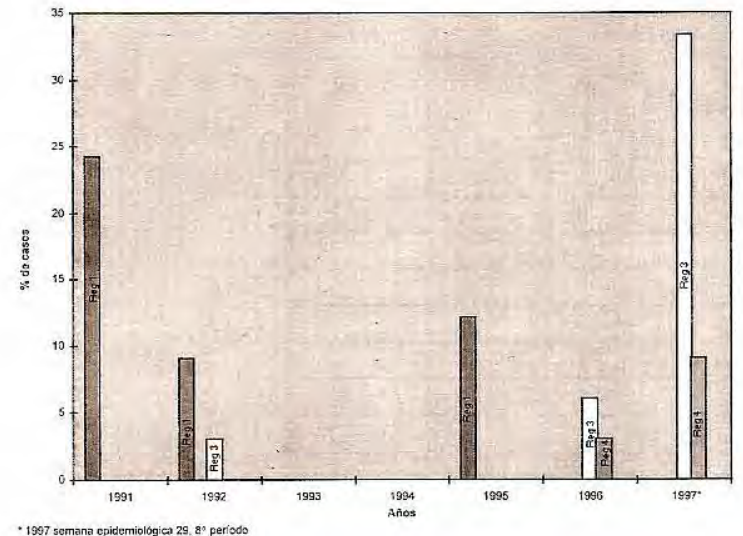

Figura 2. Defunciones por año (\%) en las regionales de Bolivar.

nes ocurridas entre 1991y 1995, $(\mathrm{p}<0,01 ; \mathrm{Rr}=4,71)$, $1996(p<0,005 ; R r=8,2), 1997(p<0,005 ; R r=11,4)$ lo cual no se observó en $1992(p>0,1)$.

\section{Discusión}

El resurgimiento del cólera se ha acompañado con la aparición de cepas emergentes y el desplazamiento de cepas endémicas en el transcurso de las pandemias, como ya ha sido descrito por algunos autores $(6,7)$.

Dalsgaard y col. (7) señalan que, en Perú, más de 95\% de los aislamientos clínicos en 1991 fueron V. cholerae 01, serotipo Inaba, y desde 1992, el serotipo Ogawa comenzó a hacerse predominante, encontrándose en más de $90 \%$ de los aislamientos de 1995.

En Colombia, Castañeda y col. (19) informaron que las cepas toxigénicas de $V$. cholerae 01 recibidas en el INS durante 1991 y 1992 , fueron en su mayoría $(99,13 \%)$ V. choleraeO1, serotipo Inaba, con una pequeña proporción $(0,87 \%)$ del serotipo Ogawa.

En nuestro medio, el desplazamiento de $V$. cholerae 01, serotipo Inaba, predominante en 1991, por el serotipo Ogawa, se hizo evidente en 1995, lo cual se explica porque desde 1992 el serotipo Ogawa aparecía discretamente en algunas regiones del país.

Los factores que determinan la emergencia y predominio de clones particulares de V. cholerae 1 toxigénico a través de la selección natural no es clara. Sin embargo, gracias al uso de métodos fenotípicos y nuevos métodos de tipificación usando la biología molecular, se está avanzando en el estudio de la epidemiología de $V$. cholerae $\mathrm{O} 1$ en Latinoamérica.

Con relación a la magnitud de la epidemia de cólera analizada en el presente estudio, se observa que la enfermedad causó mayor impacto en 1991 informándose nueve veces más casos que en 1995. Sin embargo, es preocupante que hasta el $8^{\circ}$ período de 1997 el número de casos aumentó 45,2\% con relación a los dos últimos años y la mortalidad se incrementó 4,3 veces en el último año.

Es importante señalar que, aparte de las condiciones geográficas y de los recursos naturales que marcan diferencias en las zonas o regiones del departamento, existen factores sociales comunes como son, entre otros, la deficiencia o carencia de suministro de agua potable y sistema de alcantarillado en veredas y sectores marginales de municipios en el norte y centro, la depresión momposina y el sur de Bolívar; a esa situación se debe agregar el fenómeno de los desplazados por la violencia que infortunadamente se ha extendido últimamente en más de una zona del departamento.

Al analizar el comportamiento de la enfermedad en las diferentes regionales, se observa en primera instancia que, cuando se inició la epidemia en 1991 la regional uno fue de las más afectadas y Cartagena, uno de los municipios con más casos informados, seguido de otros aledaños tales como Mahates, María la Baja, Arjona, Turbaná, Calamar, Santa Catalina, Turbaco, Villanueva, Soplaviento y Santa Rosa; mientras que Clemencia, San Estanislao y San Cristóbal estuvieron libres de cólera.

Entre otros aspectos relevantes de señalar, vale la pena mencionar que la enfermedad desapareció de los municipios del departamento de 1992 a 1994, excepto en Cartagena donde se presentaron casos. Durante el brote de 1995, la población de Mahates presentó 2,4 veces más casos que Cartagena y, además, la enfermedad apareció por primera vez en el municipio de San Estanislao; en el actual período, se observa disminución de los casos de cólera en esta regional.

En relación con Cartagena, donde el índice del cólera ha disminuido en los dos últimos años, es 
importante anotar que, por'su condición de puerto marítimo, la influencia delicanal del Dique, la carencia de infraestructura de servicios públicos en barrios marginales y los corregimientos o las veredas aledañas, además de la gran apetencia por pescados y otros productos del mar, se debe estar muy alerta teniendo en cuenta que los factores de riesgo aún permanecen.

Vemos cómo, en países industrializados, informes recientes (20) señalan casos esporádicos de có"lera en ciudades costeras como Los Angeles, a partir de productos marinos importados de la región asiática.

En la regional dos, en 1992, hubo 2,7 veces más casos que el año anterior cuando se inició la epidemia, siendo Carmen de Bolívar y Zambrano los municipios más afectados; la enfermedad alcanzó también El Guamo, San Jacinto, Córdoba y San Juan Neponuceno; este último fue la única población con casos de cólera en 1993.

Desde 1996, cuando la enfermedad apareció nuevamente en esta regional, el número de casos fue menor que en los años anteriores y Zambrano fue el único de los seis municipios con casos informados en 1997. A los problemas de salubridad en los municipios afectados, se suma la migración de campesinos por la violencia de los Montes de María; recordemos también que por esa zona circula una amplia población de viajeros especialmente en Carmen de Bolívar y la población ribereña de Zambrano.

En la regional tres, se observa que mientras la enfermedad alcanzó sólo tres de los ocho municipios durante la primera epidemia, cuando apareció de nuevo en esa zona en 1996 y hasta el período actual, además del incremento de casos, todos los municipios fueron afectados. En 1997, por ejemplo, el mayor número de casos y el más alto índice de letalidad se presentó en la pobiación de Montecristo donde la enfermedad apareció por primera vez. Otras poblaciones tales como Pinillos, Magangué, Hatillo de Loba y San Martín de Loba estuvieron entre las más afectadas.

En la regional cuatro, se observa que mientras en Mompox, Talaigua Nuevo y San Fernando hubo cólera en 1991, en Cicuco, Margarita y Tiquisio
Nuevo no se informó ningún caso; la enfermedad desapareció por cuatro años en todos los municipios, excepto en Tiquisio Nuevo y en el actual período alcanzó Cicuco, Mompox y Talaigua Nuevo, siendo estos últimos los más afectados.

Esta regional fue la tercera más afectada, aun cuando no sufre en la misma magnitud por problemas de orden público, como las regionales tres y cinco. Aparte de las precarias condiciones de salubridad de algunos de sus municipios y veredas, muy seguramente la dificultad para tener acceso a los servicios de salud contribuye a la endemicidad del cólera en esa zona.

En la regional cinco, se informaron más casos de cólera en 1991, siendo Morales y Río Viejo los más afectados; al año siguiente, alcanzó únicamente a Simití y tres años después el cólera desapareció. A partir de entonces, la disminución de la enfermedad se ha hecho evidente puesto que desde 1996 es la regional con menos casos informados de cólera .

En resumen, el cólera sigue latente en nuestro medio y los factores de riesgo para la adquisición y diseminación de la enfermedad persisten, por lo cual, además de la vigilancia epidemiológica puntual, se deben mejorar las condiciones de saneamiento ambiental y seguridad social en los sitios más vulnerables; es conveniente también orientar estudios hacia el conocimiento del perfil molecular de las cepas circulantes, sin descuidar la exploración de fuentes no convencionales de contaminación en el ambiente.

\section{Conclusiones}

En el departamento de Bolívar, 38 de 42 (90,5\%) municipios fueron afectados por el cólera entre septiembre de 1991 y el $8^{\circ}$ período de 1997 , siendo la población mayor de 14 años la más afectada con $79,2 \%$ de casos, de los cuales $60,2 \%$ fueron varones y $39,8 \%$ mujeres.

Las regionales uno y tres fueron las más afectadas con $94,5 \%$ de casos, seguidas en su orden de las regionales cuatro, dos y cinco. Cartagena fue el municipio con más casos seguido de Mahates, Montecristo, María La Baja, Pinillos, Achí, Magangué, Arjona, Turbana, Calamar, Mompox, Santa Catalina, Hatillo de Loba, Turbaco, 
San Martín de Loba, Carmen de Bolívar, Villanueva, Zambrano, Morales, Talaigua Nuevo y Río Viejo, entre otros.

Los casos de cólera ocurridos durante la epidemia de 1991 fueron nueve veces mayor que los informados en el brote de 1995 y, en el período actual, la enfermedad se incrementó $45,2 \%$ veces con relación a los últimos dos años.

El índice general de letalidad fue 0,95\%. En 1991, estuvo en $0,4 \%$ y se incrementó mostrando índices preocupantes de $0,7 \%, 1,7 \%, 3 \%$ y $4,2 \%$ en 1992, 1995, 1996 y 1997, respectivamente. En algunos municipios, la letalidad alcanzó $7 \%$.

\section{Agradecimientos}

A Armando Morales, docente del Departamento de Medicina Social, Facultad de Medicina, Universidad de Cartagena, por la asesoría estadística; José María Espinosa y José Fernando Ramos, coordinadores, Sección de Informática, Area de Zaragocilla, Universidad de Cartagena, por el apoyo tecnológico; y a Sofía Meneses, Rosalío Hernández, José M. Guardo, estudiantes del Posgrado de Microbiología, 1992, Universidad de Cartagena, por la colaboración en el procesamiento de muestras durante su entrenamiento en bacteriología.

\section{Referencias}

1. McLaughlin JC. Vibrio. In: Murray PR, Baron EJ, Pfaller MA, Tenover FC, Yolken RH, editores. Manual of clinical microbiology, $6^{\text {th }}$ ed. Washington, D.C.: ASM Press; 1995:465-76.

2. Guzmán M. Cólera. En: Chalem F, Escandón J, Campos J, Esguerra R, editores. Medicina Interna II, 3a. ed. Santa Fe de Bogotá: Impreandes Presencia; 1997:71823.

3. OPS/OMS. Actualización: situación del cólera en las Américas. Boletín Epidemiológico 1991;12(3):11-2.

4. OPS/OMS. Antecedentes históricos del cólera en las Américas. Boletín Epidemiológico 1991;12(1):10-2.

5. Freeman BA. El cólera. En: Freeman BA, ed. Microbiología de Burrows. 22 ed. México: Interamericana; 1986:551-64.
7. Faruque $\mathbf{S}$, Ahmed $\mathbf{K}$, Abdul $\mathbf{R}$, Qadri $\mathbf{F}$, et al. Emergence of a new clone of toxigenic Vibrio cholerae O1, biotype El Tor, displacing $V$. chloerae O139 Bengal in Bangladesh. J Clin Microbiol 1997;35:624-63.

8. Dalsgaard A, Albert JM, Taylor DN, Shimada T, et al. Characterization of Vibrio cholerae non-O1 serogroup obtained from an outbreak of diarrhea in Lima, Peru. J Clin Microbiol 1995;32:2725- 9.

9. Concha A. Cólera en Colombia. Temas Microbiológicos, Universidad de Antioquia 1993;14(3):1-12.

10. OPS/OMS. El cólera en las Américas. Actualización. Boletin epidemiológico 1991;12(2):1-10.

11. Rivas F, de la Hoz F. Algunos aspectos clínicos del cólera en el Hospital San Andrés de Tumaco. Biomédica 1992;12:102-8.

12. Mendoza M, Carmona R. Cólera grave en el Hospital Universitario de Cartagena. Acta Médica Cartagena 1997;5:133-7.

13. Castañeda E, Chinchilla M, de la Hoz F. Cólera en Colombia: estudio seroepidemiológico, 1994. Acta Méd Colomb 1994;19(Supl):253.

14. Mattar S. Características del Vibrio Cholerae 01, EI Tor, serotipo Inaba, aislado en la epidemia del cólera en Cartagena, Colombia. Enf Inf y Microbiol Clin 1992; 10(8):525-30.

15. DANE. Población ajustada 1993 y proyecciones preliminares por cabecera y resto según municipios a junio 30 de 1995-1997. Dirección Técnica de Censo, Departamento de Bolívar.

16. CDC/NCID. Métodos de laboratorio para el diagnóstico de Vibrio cholerae (edición en español). Washington, D.C.: OPS; 1994:17-72.

17. Ministerio de Salud/Instituto Nacional de Salud. Cólera. Serie de notas e informes técnicos No.19;. 1991:13-47.

18. Waschmut IK, Morris GK, Freely JC. Vibrio. En: Lennette E, Balows A, Hausler W, Truant JP, editores. Manual de microbiología clínica, 3a. ed. Buenos Aires: Editorial Médica Interamericana; 1982:286-90.

19. Dalsgaard A, Skov MN, Seritchantalergs O, Echeverría P, Meza R, et al. Molecular evolution of Vibrio cholerae 01 strains isolated in Lima, Peru. J Clin Microbiol 1997;35:1151-6.

20. Castañeda E, Muñoz N, Vargas Cl de, et al. Diagnóstico bacteriológico del cólera. Actividades del Laboratorio Nacional de Referencia. Biomédica 1992; 12:131-6.

21. Vugia DJ, Shefer AM, Douglas J, Grene KD, et al. Cholera from the Philippines to California. J Clin Microbiol 1997;35:284-5. 